\title{
Antibacterial properties of silver nanoparticles in three different sizes and their nanocomposites with a new waterborne polyurethane
}

This article was published in the following Dove Press journal:

International Journal of Nanomedicine

18 November 2010

Number of times this article has been viewed
Abstract: Silver nanoparticles (AgNPs) are strong bactericidal agents but they are also cytotoxic. Embedding them in a polymer matrix may reduce their cytotoxic effect. In the present study, AgNPs in three average sizes were tested for their antibacterial activities and cytotoxicity. Nanocomposites from a new waterborne polyetherurethane (PEU) ionomer and AgNPs were prepared without the use of any crosslinker. It was observed that the antibacterial activity of AgNPs against Escherichia coli started at the effective concentration of 0.1-1 ppm, while that against Staphylococcus aureus started at higher concentrations of 1-10 ppm. Cytotoxicity of AgNPs was observed at the concentration of $10 \mathrm{ppm}$. AgNPs with smaller average size showed greater antibacterial activity as well as cytotoxicity. The PEU synthesized in this study showed high tensile strength, and the addition of AgNPs at all sizes further increased its thermal stability. The delicate surface features of nanophases, however, were only observed in nanocomposites with either small- or medium-sized AgNPs. PEU-Ag nanocomposites had a strong bacteriostatic effect on the growth of E. coli and S. aureus. The proliferation of endothelial cells on PEU-Ag nanocomposites was enhanced, whereas the platelet adhesion was reduced. The expression of endothelial nitric oxide synthase gene was upregulated on PEU-Ag containing small-sized AgNPs (30 ppm) or medium-sized AgNPs (60 ppm). This effect was not as remarkable in nanocomposites from large-sized AgNPs. Overall, nanocomposites from the PEU and $60 \mathrm{ppm}$ of the medium-sized ( $5 \mathrm{~nm}$ ) AgNPs showed the best biocompatibility and antibacterial activity. Addition of smaller or larger AgNPs did not produce as substantial an effect in PEU, especially for the larger AgNPs.

Keywords: silver nanoparticles, polyurethane, antibacterial activity, biocompatibility

\section{Introduction}

Bulk silver possesses relatively good biocompatibility. Silver nanoparticles (AgNPs) on the other hand show outstanding antibacterial properties. Many investigations have focused on their bactericidal effect and applications in the plastics, health, textile, and paint industries. ${ }^{1,2}$ For example, AgNPs coated on a water filter are resistant to washing and could serve to remove bacteria from water. ${ }^{3} \mathrm{AgNPs}$ mixed with bone cement show high antibacterial activity against many strains. ${ }^{4}$ However, enthusiasm for AgNPs has been hampered by their cytotoxicity and genotoxicity. ${ }^{5}$ The in vitro cytotoxicity of AgNPs in mammalian cells has been reported. ${ }^{6}$ AgNPs may inhibit the segregation of chromosomes. ${ }^{7}$ Genotoxicity, including DNA damage and chromosomal aberrations, has also been observed in human glioblastoma cells treated with AgNPs. ${ }^{8}$

Conventional polyurethane processes involve the use of a significant amount of organic solvents. The products sometimes contain residual solvent and even free 
isocyanate monomers that are highly toxic to organisms. Because of the environmental advantages, waterborne polyurethane has gained great attention in many commercial fields, such as coatings and adhesives for various substrates. ${ }^{9-11}$ Waterborne polyurethane has many advantages relative to conventional solvent-borne polyurethane, including low viscosity at high molecular weight, and can be extensively used in many applications. ${ }^{12}$ However, a few properties of waterborne polyurethane need to be further modified for specific uses, including water resistance and thermal stability. It has been reported that addition of gold nanoparticles to waterborne polyurethane could upregulate expression of the endothelial nitric oxide synthase (eNOS) gene, ${ }^{13}$ which is an important regulator of cell proliferation and migration. When AgNPs are dispersed in waterborne polyurethane at low concentrations, thermal and mechanical properties, as well as biostability and antibacterial ability, are improved. ${ }^{14}$ More importantly, the cytotoxicity of AgNPs may be reduced if they are embedded in a polymer matrix.

Most waterborne polyurethane (polyurethane emulsion) requires reacting with a crosslinker before being cast into the final products. The crosslinker is toxic, and may react or interact with AgNPs. In this study, we synthesized a new polyether type of polyurethane (PEU ionomer) that did not require a crosslinker. We tried to optimize the size/concentration of AgNPs using AgNPs in three different sizes. The surface characteristics and cellular response (by bacteria, endothelial cells, platelets, and monocytes) of the nanocomposites were investigated.

\section{Materials and methods}

\section{Antibacterial activity of silver nanoparticles}

In this study, we used AgNPs of three different sizes, ie, 3-4 nm (small, Ag-S), 5-7 nm (medium, Ag-M) and 10-40 nm (large, Ag-L), supplied from Gold NanoTech, Taipei, Taiwan. These AgNPs were produced by physical manufacturing and did not contain any surface modifiers or stabilizers. ${ }^{15}$ The initial concentration was determined by an inductively coupled plasma mass spectrometer (Sciex Elan 6100 DRC; Perkin Elmer, Daytona Beach, FL). The sizes were confirmed by a transmission electron microscope (JEM-1200; JEOL, Tokyo, Japan) operated at $110 \mathrm{kV}$. The size distribution and average size were computed by software based on more than a hundred particles on the images. Staphylococcus aureus (ATcc6538p) and Escherichia coli (JM109) were used for the antibacterial experiments. The bacteria were activated and then collected by centrifugation. After resuspension in
$1 \mathrm{~mL}$ of phosphate-buffered saline, bacteria were diluted to $1-4 \times 10^{5}$ colony-forming units $(\mathrm{CFU}) / \mathrm{mL}$. After incubation at $35^{\circ} \mathrm{C}$ for 24 hours, AgNPs with different concentrations were laid on agar. After further incubation for 18 hours, the bacterial colonies were counted. Double-distilled water served as the control. $\mathrm{AgNO}_{3}$ of the same concentration was included for comparison.

\section{Cytotoxicity of silver nanoparticles}

L929 (NCTC Clone 929) mouse skin fibroblasts were cultured in low-glucose Dulbecco's modified Eagle's medium (Gibco, Tulsa, OK) containing 10\% fetal bovine serum. An initial density of $5 \times 10^{4}$ cells were seeded in each well of a 24-well tissue culture plate. After 24 hours of incubation, the medium was replaced by fresh medium containing AgNPs at final concentrations of 1 and $10 \mathrm{ppm}$. The treatment containing only culture medium was used as the negative control, while that containing culture medium and 5\% dimethyl sulfoxide was applied as the positive control. The cells were cultured in an incubator at $37^{\circ} \mathrm{C}$ and $5 \% \mathrm{CO}_{2}$ for 24 hours. After treatment, the adherent cells were trypsinized, centrifuged, and resuspended. The number of the cells was counted by a hemocytometer under an inverted phase contrast microscope (TE-300; Nikon, Tokyo, Japan).

\section{Preparation of waterborne polyetherurethane and polyesterurethane}

Two types of polyurethane were synthesized in this study. The schematic diagram for preparing the new waterborne PEU ionomer is shown in Figure 1. Bis(hydroxymethyl) propionic acid (DMPA; Fluka, St. Louis, MO) and the macrodiol poly(tetramethylene oxide) (PTMO) were mixed at $50^{\circ} \mathrm{C}$ in methyl ethyl ketone (Fluka). After complete dispersion, dicyclohexylmethane diisocyanate $\left(\mathrm{H}_{12} \mathrm{MDI}\right.$; Bayer, Pittsburg, PA) and 4,4-diphenylmethane diisocyanate (MDI; Bayer) were added to react at $75^{\circ} \mathrm{C}$. Triethylamine (TEA; $\mathrm{RDH}$, Chicago, IL) was then added after the previous reaction was finished. Distilled water was introduced and isophorone diamine (IPDA) was finally added to complete the synthesis of PEU emulsion (with 30\% solid content in distilled water). The molar ratio of DMPA: PTMO: $\mathrm{H}_{12}$ MDI: MDI: TEA: IPDA was 1.2:1:2.33:1.4:1.2:1.53.

Waterborne polyesterurethane (PSU) that required a crosslinker was prepared for comparison purposes according to the method previously reported in the literature. ${ }^{16}$ Briefly, the PSU emulsion (with 50\% solid content in distilled water) was synthesized using hexamethylene diisocyanate and the macrodiol poly(butadiene adipate, average molecular 


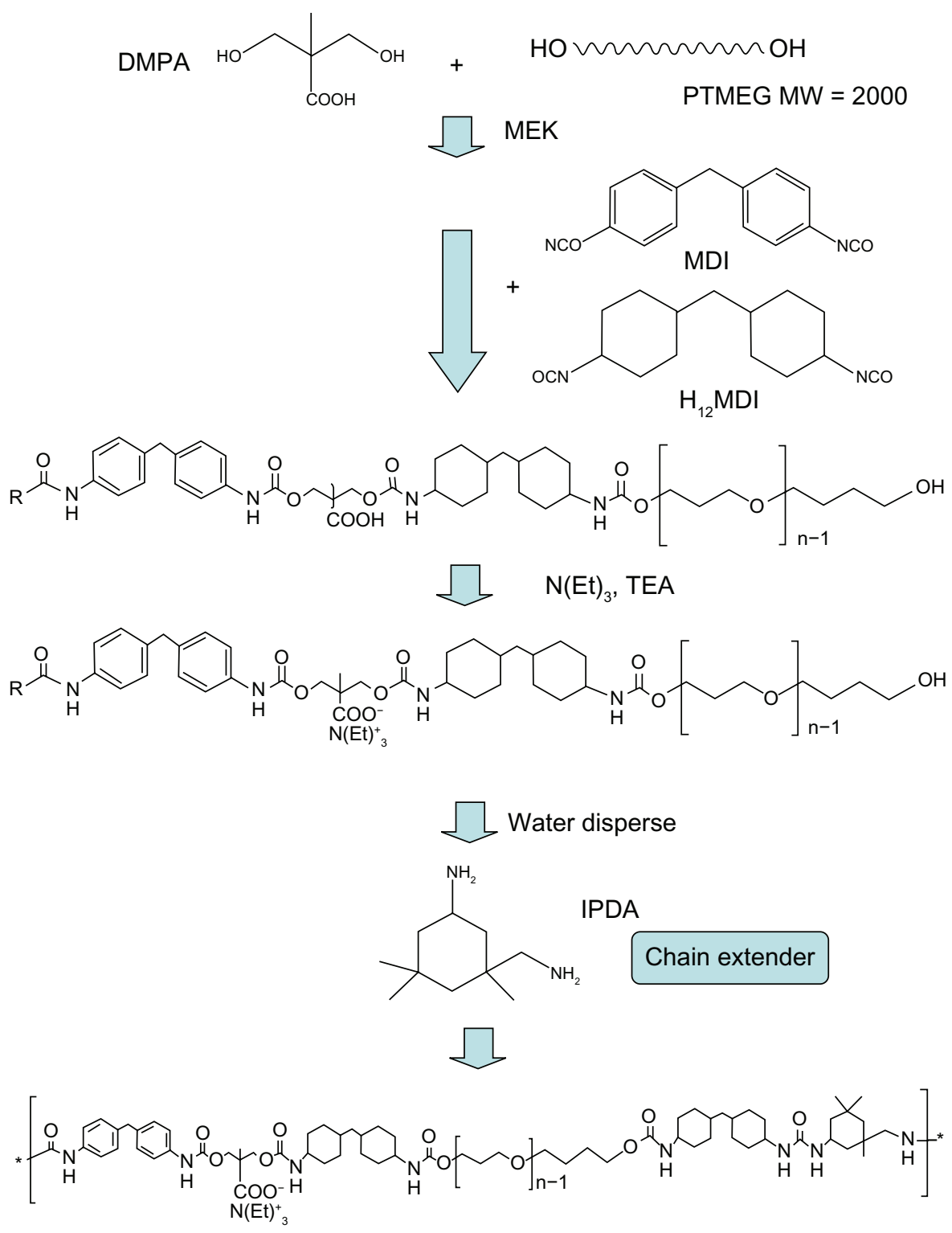

Figure I Schematics for the synthesis of waterborne polyetherurethane ionomer.

weight 2000) at a molecular ratio 3:1 and chain-extended by ethylene diamine sulfonate sodium salt and ethylene diamine. The crosslinker was a mixture of the isocyanurate trimer of hexamethylene diisocyanate and 6\% Bayer hardener (made from the isocyanurate trimer of hexamethylene diisocyanate and polyethylene glycol). PSU was prepared by mixing the emulsion with the crosslinker ( $1 \%$ of the total weight).

To make thin membranes used for biologic tests, $10 \%$ polyurethane emulsion (PEU emulsion or PSU emulsion/ crosslinker) was loaded onto coverslip glass (15 mm diameter; Matsunami, Osaka, Japan) and cast by a spin coater (PM-490; Synrex, Taipei, Taiwan) at $2300 \mathrm{rpm}$ for 30 seconds. Thick membranes for other tests were made by pouring polyurethane emulsion into a Teflon mold. The cast samples were dried in the vacuum at $60^{\circ} \mathrm{C}$ for 48 hours to guarantee that there was no residual water in the sample. These samples were stored in the drying cabinet for later experiments.

To obtain the mechanical properties of the two elastomers, the polyurethane specimens were cut in $20 \mathrm{~mm} \times 1 \mathrm{~mm}$ (length $\times$ width) samples and tested by a universal testing instrument (HT-8504; Hung Ta, Taipei, Taiwan). The Young's modulus, tensile strength, and elongation were obtained at a constant speed $(1 \mathrm{~m} / \mathrm{min})$.

\section{Fabrication of PEU-Ag nanocomposite films}

The PEU emulsion was adjusted to $10 \%$ solution (w/w) by distilled water or by water suspension of AgNPs. The mixture 
was then sonicated for 30 minutes. The prepared emulsion and their cast films either contained no AgNPs or contained AgNPs (PEU-Ag). The PEU-Ag films were divided into three groups based on their silver particle sizes, including PEUS (PEU mixed with small-sized AgNPs), PEUM (PEU mixed with medium-sized AgNPs), and PEUL (PEU mixed with large-sized AgNPs). The final concentrations of AgNPs in dried PEU were 0, 15, 30, 60, 120, and 240 ppm. Nanocomposite films were prepared following the same procedure for fabricating the polyurethane films as described earlier.

\section{Characterization of PEU-Ag nanocomposites}

Thermogravimetric analysis of PEU-Ag nanocomposites was performed with a thermogravimetric analyzer (TGA2050; TA Instruments, New Castle, DE). The pyrolytic temperatures ( $\mathrm{T}_{\text {onset }}$ and $\mathrm{T}_{\mathrm{p}}$ ) were determined by the thermogravimetric analysis curves. The glass transition temperature $\left(\mathrm{T}_{\mathrm{g}}\right)$ was determined using a dynamic scanning calorimeter (SSC/5200; Seiko, Tokyo, Japan).

The surface morphology of PEU-Ag nanocomposites was examined by an atomic force microscope (CP-II; Veeco, Plainview, NY). The topography and phase images were obtained in the tapping mode in air with a triangular cantilever (force constant of 20-80 N/m) supporting an integrated pyramidal tip of phosphorus-doped silicon (RTESPA-CP, Veeco). The surface zeta potential of PEU-Ag films was determined by a zeta potential and particle size analyzer (Delsa Nano S; Beckman Coulter, Osaka, Japan).

\section{Antibacterial activity of PEU-Ag nanocomposites}

The protocol to measure the antimicrobial activity of PEUAg nanocomposites followed the JIS 2801 standard for microbiostasis of film-type materials. The test bacteria were $S$. aureus and E. coli. These bacteria were cultured in broth for 24 hours. The broth was prepared by peptone (Himedia, Mumbai, India), beef extract (Himedia) and $\mathrm{NaCl}$ (Sigma, St Louis, MO) dissolved in distilled water and sterilized by an autoclave at $103 \mathrm{kPa}$ for 20 minutes. Film samples were cut to squared shape $(5 \mathrm{~cm} \times 5 \mathrm{~cm})$. The samples were placed in $9 \mathrm{~cm}$ Petri dishes after sterilization by $70 \%$ ethanol and washed twice with distilled water. The bacteria were cultured overnight and diluted to $2.5-10 \times 10^{5} \mathrm{CFU} / \mathrm{mL}$ by diluted broth. The diluted broth was the broth diluted from the nutrient broth $(1 / 500)$ by purified water. The inoculum was $0.4 \mathrm{~mL}$ bacterial suspension and placed on the surface of each sample. In order to spread inoculum solution uniformly, a polyethylene film was used to cover the sample surface. The plain PEU samples were used as the control material and were separated into two parts, ie, the immediate and the tested groups. The immediate control group was performed by immersion of the specimen containing the covered film immediately after the inoculation. The digestion in a sterilized tube with $10 \mathrm{~mL}$ soybean-casein digest broth (Becton, Dickinson and Company, Franklin Lakes, NJ) and lecithin polysorbate (MP Biomedicals, Santa Ana, CA) was carried out in an ultrasonic bath. All other samples of plain PEU or nanocomposites in Petri dishes were inoculated with the test bacteria and incubated for 24 hours under a relative humidity of not less than $90 \%$ and a temperature of $35^{\circ} \mathrm{C}$. These samples were treated using the same procedures as the immediate control group. The digestive solution containing bacteria was serially diluted and plated on agar plates. The colony growth was counted and the microbiostasis ratio was calculated as:

[CFU in immediate group of control - CFU in tested group of control or CFU of the samples/CFU of immediate control group of control] $\times 100 \%$

\section{Cell attachment and proliferation on PEU-Ag nanocomposites}

Bovine carotid arterial endothelial cells were purchased from the American Type Culture Collection. These cells can be cultured for higher passages without appreciable loss of phenotype or growth rate. Cells from 15-20 passages were used in this study. Endothelial cells were cultured in low-glucose Dulbecco's modified Eagle's medium supplemented with 1\% fetal bovine serum, $2 \mathrm{mM}$ glutamine and $1 \%$ (v/v) antibiotics (10000 U/mL penicillin $\mathrm{G}$ and $10 \mathrm{mg} / \mathrm{mL}$ streptomycin). Polyurethane and nanocomposites coated on coverslip glass were individually sterilized by $70 \%$ ethanol, washed using phosphate-buffered saline, and placed in each well of the 24-well tissue culture plates. Cells cultured in a blank well (tissue culture polystyrene, TCPS) were used as the control. One $\mathrm{mL}$ of cell suspension containing $2 \times 10^{4}$ cells was placed into each well of the plate. After 72 hours of incubation, the adherent cells were trypsinized, and their number was counted under the inverted microscope. In addition, the viability of adherent cells was determined by MTT assay. For the MTT assay, $300 \mu \mathrm{L}$ 3-(4,5-dimethylthiazol-2-yl)-2, 5 -diphenyltetrazolium bromide solution $(0.5 \mathrm{mg} / \mathrm{mL}$, $1 \times$ phosphate-buffered saline) was added. After four hours of incubation at $37^{\circ} \mathrm{C}$ in the dark, the supernatant was removed and $600 \mu \mathrm{L}$ dimethyl sulfoxide was added into each well to dissolve the resulting formazan crystals. A microplate reader 
(Molecular Devices, Sunnyvale, CA) was used to measure the optical density at wavelength $550 \mathrm{~nm}$, which represented cell viability on the surface of the samples.

\section{RNA extraction and real-time polymerase chain reaction analysis}

The total RNA from endothelial cells was extracted by the Trizol $^{\circledR}$ reagent (Invitrogen, Carlsbad, CA) following the manufacturer's instructions. cDNA was synthesized from $1 \mu \mathrm{g}$ of total RNA using RevertAid ${ }^{\mathrm{TM}}$ First Strand cDNA synthesis kit (MBI Fermentas, St. Leon-Rot, Germany). The aliquot of cDNA was amplified by polymerase chain reaction (PCR) using the PCR Master Mix Kit (Applied Biosystems, Foster City, CA). The mixture of PCR reaction was $25 \mu \mathrm{L}$ containing $10 \mathrm{mM}$ targeted gene oligonucleotide primers. Gene expression of eNOS and GAPDH (internal control) was performed by PCR consisting of denaturing at $94^{\circ} \mathrm{C}$ for 30 seconds, annealing at $52^{\circ} \mathrm{C}$ for 30 seconds, and extension at $72^{\circ} \mathrm{C}$ for 50 seconds with 35 cycles using a Thermal Cycler (GeneAmp PCR System 2700; Applied Biosystems). The primer sequence used for eNOS was $5^{\prime}$ ATAGAATTCACCAGCACCTTTGGGAATGGCGAT3' (forward) and 5' ATAGAATTCGGATTCACTGTCTGTGTTGCTGGACTCCTT 3' (reverse), and for GAPDH was 5'TCCCTCAAGATTGTCAGCAA3' (forward) and 5' AGATCCACAACGGATACATT3' (reverse). Semiquantification of eNOS/GAPDH ratio was conducted by densitometric analysis (LabWork Image Acquisition and Analysis software; Applied Biosystems).

\section{Platelet adhesion on nanocomposites}

For platelet adhesion, $0.5 \mathrm{~mL}$ platelet-rich plasma $\left(\sim 2 \times 10^{6}\right.$ platelets $/ \mu \mathrm{L}$, obtained from the Chinese Blood Foundation, Taiwan) was added on the surface of testing material in a 24-well culture plate. The testing material was gently washed with HEPES-buffered saline after incubation for one hour. The adherent platelets in one of the duplicates were counted by a blood cell counter (KX-21; Sysmex, Kobe, Japan) after treatment with trypsin. ${ }^{17}$

\section{Monocyte response to nanocomposites}

Human blood monocytes were obtained from the venous blood of unmediated donors and isolated by density gradient centrifugation using Percoll (Sigma). ${ }^{17}$ The protocol was approved by the Institutional Review Board. The medium of RPMI-1640 (Gibco) containing 10\% fetal bovine serum and $1 \%(\mathrm{v} / \mathrm{v})$ antibiotics $(10000 \mathrm{U} / \mathrm{mL}$ penicillin $\mathrm{G}$ and $10 \mathrm{mg} / \mathrm{mL}$ streptomycin) was used to suspend the monocytes, and the concentration was adjusted to $1 \times 10^{5}$ cells $/ \mathrm{mL}$. The $1 \mathrm{~mL}$ aliquot of the monocyte suspension was added to the surface of nanocomposites placed in a 24-well culture plate. After incubation for 96 hours, cells were treated by trypsin and counted by a cell counter (Multisizer 3; Beckman Coulter). The numbers of cells including nonactivated monocytes and activated monocytes (macrophages) were determined based on their sizes (6-9 $\mu \mathrm{m}$ for nonactivated monocytes and 9-25 $\mu \mathrm{m}$ for activated macrophages). The percent activation of monocytes was calculated as the ratio of the number of adherent macrophages and the sum of monocytes and macrophages. ${ }^{17}$

\section{Statistical analysis}

The values were expressed as the mean \pm standard deviation. Three similar experiments were performed for each type of experiment in this study. The statistical differences among the experimental groups were determined by analysis of variance using the Student's $t$-test. $P$ values $<0.05$ were considered statistically significant.

\section{Results}

The average particle size of AgNPs used in this study was $3.08 \pm 1.16 \mathrm{~nm}, 5.75 \pm 1.12 \mathrm{~nm}$, and $24.85 \pm 6.06 \mathrm{~nm}$ for Ag-S, Ag-M, and Ag-L, respectively, based on transmission electron microscopy image analysis.

The antibacterial activity of AgNPs (Ag-S, Ag-M, and Ag-L) against E. coli and $S$. aureus is shown in Figure 2. AgNPs of all sizes showed excellent antibacterial effects. The antibacterial activity against $E$. coli started about $0.1 \mathrm{ppm}$, while that against $S$. aureus started at about $1 \mathrm{ppm}$, ie, a 10-fold increase. At these critical concentrations, the antibacterial activity against $E$. coli could be distinguished among Ag-S, Ag-M, and Ag-L. The smaller the average size of the AgNPs, the greater the antibacterial effect observed. Silver ions on the other hand were strongly bactericidal at a concentration of $0.1 \mathrm{ppm}$.

The cytotoxicity of AgNPs is shown in Figure 3. Significant cytotoxicity was detected at higher concentrations (10 ppm) of small-sized AgNPs, but not at $1 \mathrm{ppm}$. Silver ions at both concentrations ( 1 and $10 \mathrm{ppm}$ ) showed marked cytotoxicity. Ag-M and Ag-L at 10 ppm, although it killed all bacteria, did not show significant cytotoxicity.

The tensile properties of the synthesized PEU and PSU are shown in Table 1. Based on the result, the Young's modulus of PEU and the previously reported PSU was similar. However, the tensile strength was higher for PEU. The attachment and proliferation of endothelial cells seeded 
A

\section{E. coli}

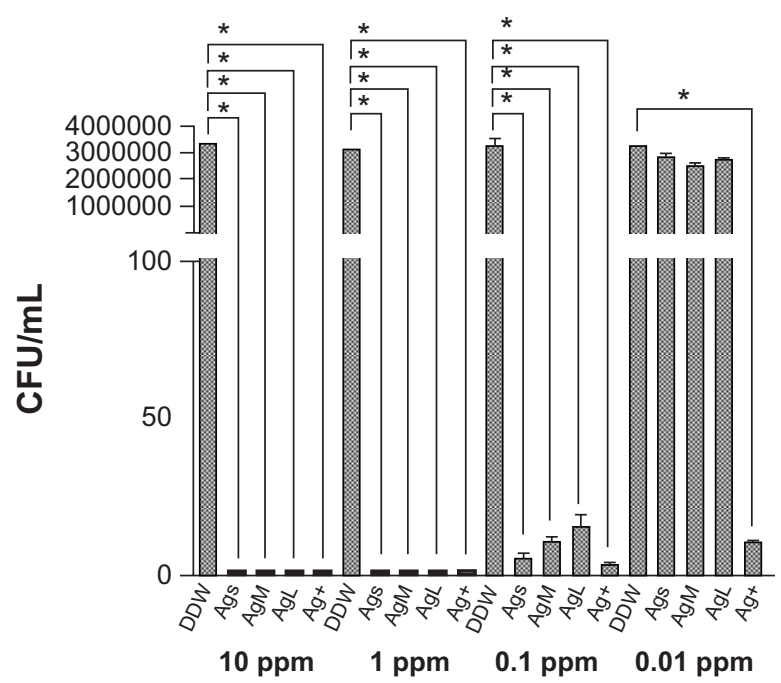

B

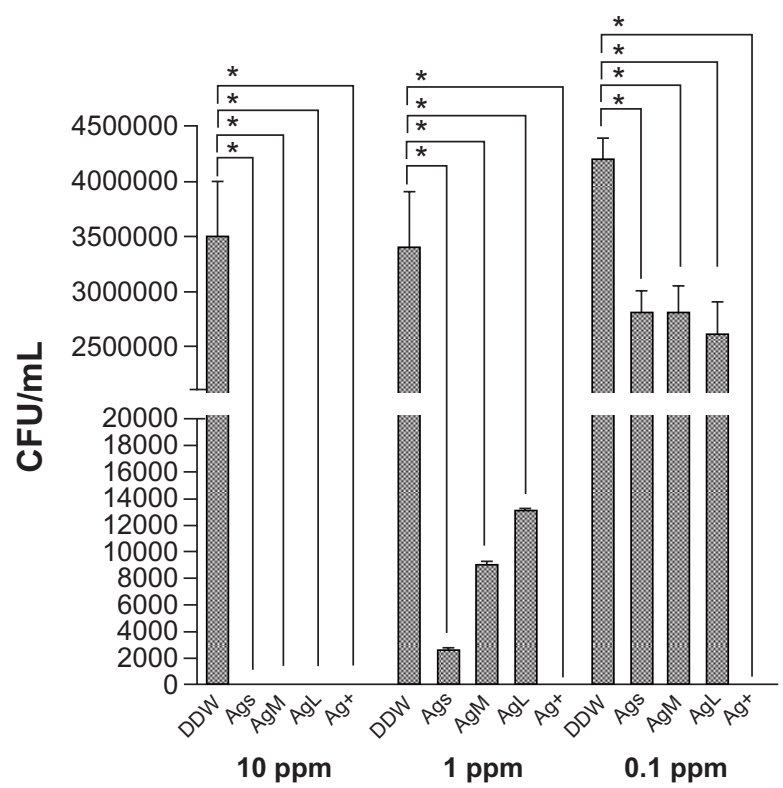

Figure 2 Antibacterial activity of silver nanoparticles against A) Escherichia coli and B) Staphylococcus aureus, shown as bacterial colony-forming units after exposure to silver nanoparticles of different sizes and concentrations for 24 hours. Doubledistilled water served as the control. Silver ion $\left(\mathrm{Ag}^{+}\right)$of the same concentration was included for comparison.

Note: $* P<0.05$ relative to double-distilled water.

on PEU and PSU are shown in Figure 4. The attachment of endothelial cells on both PU was comparable at 24 hours. The number of cells on PSU was a little higher than that on PEU after 72 hours.

The thermal stability of the PEU-Ag nanocomposites is shown in Table 2. The $\mathrm{T}_{\text {onset }}$ of PEU $\left(238^{\circ} \mathrm{C}\right)$ increased up to $244^{\circ} \mathrm{C}$ in PEU-Ag. $\mathrm{T}_{\mathrm{p}}$ increased from $402^{\circ} \mathrm{C}$ to $404^{\circ} \mathrm{C}$. $\mathrm{T}_{\mathrm{g}}$ slightly decreased from $-72.3^{\circ} \mathrm{C}$ to $-73.7^{\circ} \mathrm{C}$, indicating

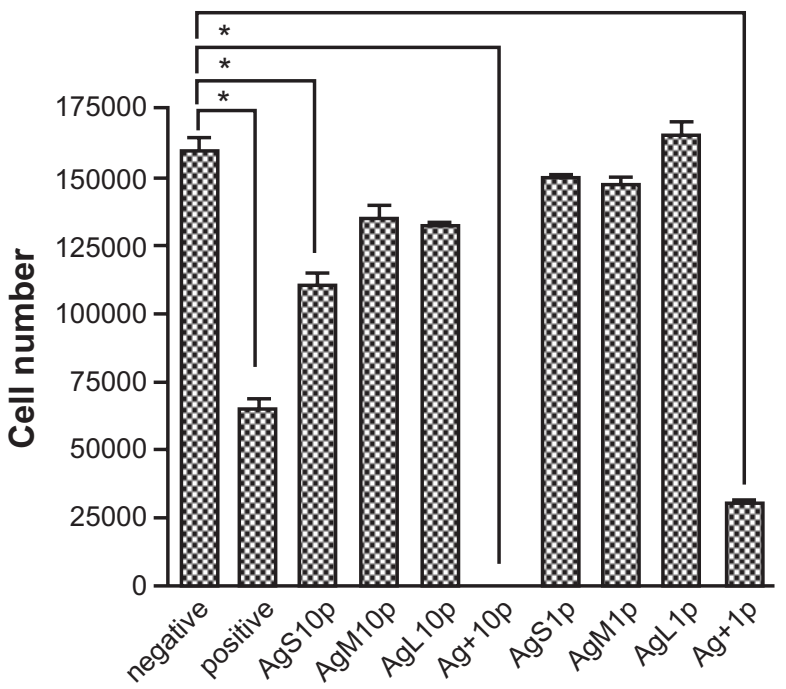

Figure 3 Cytotoxicity of silver nanoparticles for L929 fibroblasts. The cells were incubated in culture medium containing silver nanoparticles (of different sizes) at the concentrations of I ppm (I P) and 10 ppm ( 10 p) for 24 hours.

Note: $* P<0.05$ relative to negative control.

more microphase separation between the soft and hard segments of the bulk materials.

Figure 5 presents the phase diagrams of PEU mixed with different sizes of AgNPs at different prepared concentrations. The bright area indicated the hard segment, whereas the dark area indicated the soft segment. The surface microstructure of PEU changed upon addition of AgNPs. The roughness was not significantly altered. The surface microphase separation of PEU increased when the concentration of small or medium sized AgNPs increased. This phenomenon was not observed for larger AgNPs. In addition, the micellar and lamellar structure in the plain PEU changed when smaller AgNPs were added. For example, at $15-30 \mathrm{ppm}$ of small AgNPs, the soft domains (ie, darker regions in the image) were more dispersed and smaller in size, indicating a more delicate nanostructure. Beyond $60 \mathrm{ppm}$, the soft and hard domains became larger. At 15-60 ppm of medium-sized AgNPs, the soft domains were also more dispersed and the structure was more delicate as well. These delicate surface domain structures were absent in PEU when large AgNPs (ie, PEUL) were added.

The values of the surface zeta potential for PEU and PEU-Ag are shown in Table 3. All the films were negatively charged. The charge increased with the elevation of AgNP concentration, and then slightly decreased for higher concentrations of AgNPs. The most negative surface zeta potential occurred in PEU containing 30 or 60 ppm of Ag-M (ie, PEUM 30 ppm and PEUM 60 ppm). 
Table I The tensile properties of waterborne polyurethane synthesized in this study

\begin{tabular}{llll}
\hline Materials & $\begin{array}{l}\text { Young's modulus } \\
(\times \mathbf{~ m P a})\end{array}$ & $\begin{array}{l}\text { Tensile strength } \\
(\mathbf{m P a})\end{array}$ & Elongation (\%) \\
\hline Polyetherurethane & $20.7 \pm 1.8$ & $20.5 \pm 2.8$ & $368 \pm 0.22$ \\
PSU & $25.2 \pm 2.3$ & $10.5 \pm 3.4$ & $412 \pm 0.31$ \\
\hline
\end{tabular}

Note: *PSU was a waterborne polyesterurethane that required a crosslinker during synthesis and was described previously. ${ }^{16}$

The antibacterial activity of the nanocomposites is shown in Figure 6. PEU showed only weak antibacterial ability $(68 \%$ of microbiostasis ratio against $E$. coli). The microbiostasis ratio for E. coli increased to about $94 \%$ in PEU-Ag containing $30 \mathrm{ppm}$ Ag-S (ie, PEUS $30 \mathrm{ppm}$ ). Moreover, the microbiostasis ratio was about $96 \%$ for PEU-Ag containing 60 ppm Ag-M (ie, PEUM 60 ppm). The AgNP-dose dependent phenomenon of antibacterial effect was not evident for PEU-Ag against E. coli. The microbiostasis ratio of PEU was only $16 \%$ for $S$. aureus. The microbiostasis ratio for $S$. aureus was increased to above $80 \%$ for PEU-Ag containing 120-240 ppm Ag-S, 120-240 ppm Ag-M, or 240 ppm Ag-L. The antibacterial effect of PEU-Ag against $S$. aureus was in a AgNP-dose-dependent manner.

The attachment and proliferation of endothelial cells on nanocomposites are shown in Figure 7. The attachment of endothelial cells evaluated at 24 hours by either the direct cell counting or MTT assay showed no obvious difference between PEU and PEU-Ag nanocomposites. The proliferation of endothelial cells evaluated by the number of cells at 72 hours in each group was the highest for the nanocomposites containing Ag-S at $30 \mathrm{ppm}, \mathrm{Ag}-\mathrm{M}$

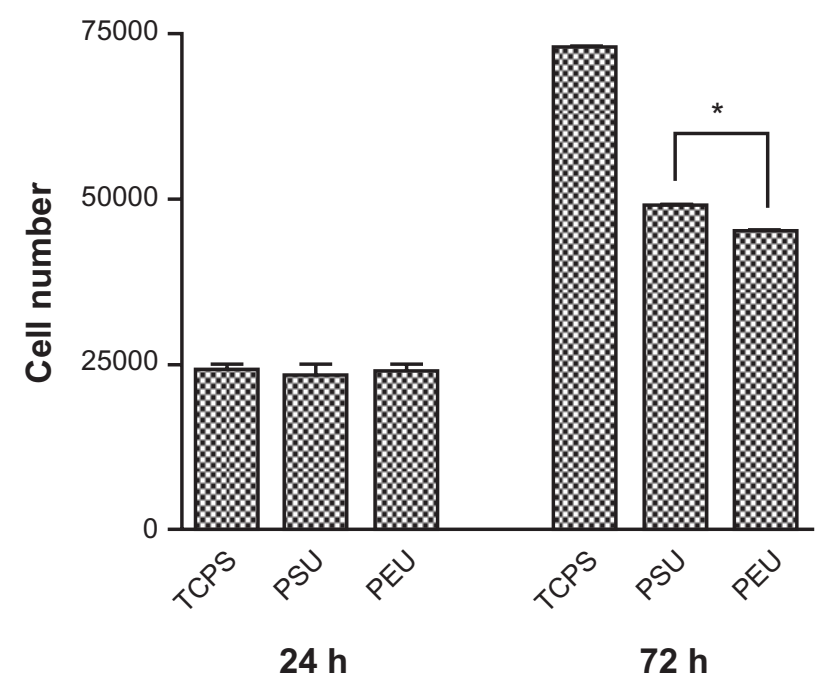

Figure 4 Proliferation of endothelial cells on the waterborne polyurethane (polyetherurethane and polyesterurethane) at 24 and 72 hours. Note: $* P<0.05$. at $60 \mathrm{ppm}$, or $\mathrm{Ag}-\mathrm{L}$ at $15 \mathrm{ppm}$. Concentrations below or above the aforementioned concentrations gave a lower cell proliferation. The highest proliferation among all groups was PEUM $60 \mathrm{ppm}$. The gene expression of eNOS at 72 hours for endothelial cells on different nanocomposites is shown in Figure 8. The eNOS gene expression was lower for cells grown on PEU than that on TCPS. The eNOS gene expression was upregulated on a few PEU-Ag nanocomposites, including PEUS 30 ppm, PEUS 240 ppm, PEUM $60 \mathrm{ppm}$, and PEUL $15 \mathrm{ppm}$. The greatest level of eNOS gene expression was observed for PEUM 60 ppm, followed by PEUS $30 \mathrm{ppm}$.

Table 4 shows the platelet adhesion on the nanocomposites, which is a way to evaluate blood compatibility in vitro. The number of platelets adhered on each plain PEU sample was about $11 \times 10^{6}$. The amount of platelet adhesion was reduced in nearly all nanocomposites, especially for PEUS $60 \mathrm{ppm}$ and PEUM $30 \mathrm{ppm}$. On the other hand, the inflammatory response, as measured by monocyte activation, did not show a dramatic difference between PEU and PEU-Ag nanocomposites. Monocyte activation was reduced from about $26 \%$ in PEU to about $22 \%$ in PEUS $15-30 \mathrm{ppm}$, PEUM $60 \mathrm{ppm}$, or PEUL $15 \mathrm{ppm}(P<0.05)$. The other nanocomposites did not show significantly different results from PEU.

\section{Discussion}

The antibacterial activity of AgNPs against E. coli was found to be higher than that against $S$. aureus, with almost one order of difference in the applied concentrations. Kim et al ${ }^{18}$ reported that the minimum inhibitory concentration of AgNPs for E. coli $(>3.3 \mathrm{nM})$ was lower than that for $S$. aureus $(>33 \mathrm{nM})$, which was a 10 -fold difference. This may be attributed to the fact that Gram-negative bacteria have relatively thin cell walls and thus are more prone to physical destruction. ${ }^{18}$ The data further showed that smaller AgNPs had higher antibacterial activity against $E$. coli and $S$. aureus. Antibacterial activity decreased with the increased size of AgNPs. This finding is in agreement with previous reports in the literature suggesting that the larger specific 
Table 2 The measurement of $T_{\text {onsett }} T_{p}$ and $T_{g}$ for polyetherurethane and polyetherurethane-silver

\begin{tabular}{llll}
\hline Materials & $\mathbf{T}_{\text {onset }}\left({ }^{\circ} \mathbf{C}\right)$ & $\mathbf{T}_{\mathbf{p}}\left({ }^{\circ} \mathbf{C}\right)$ & $\mathbf{T}_{\mathbf{g}}\left({ }^{\circ} \mathbf{C}\right)$ \\
\hline PEU & 238.0 & 402.6 & -72.3 \\
PEUSI5 & 242.6 & 402.7 & -73.3 \\
PEUS30 & 243.9 & 403.2 & -73.5 \\
PEUS60 & 243.4 & 403.0 & -73.1 \\
PEUSI 20 & 242.2 & 402.5 & -72.7 \\
PEUS240 & 238.2 & 403.1 & -72.5 \\
PEUMI5 & 242.7 & 403.7 & -73.1 \\
PEUM30 & 243.2 & 404.2 & -73.7 \\
PEUM60 & 244.1 & 404.2 & -73.3 \\
PEUMI20 & 242.3 & 403.6 & -73.0 \\
PEUM240 & 239.1 & 403.5 & -72.5 \\
PEUL15 & 242.2 & 404.3 & -73.7 \\
PEUL30 & 239.5 & 403.2 & -72.3 \\
PEUL60 & 238.5 & 403.4 & -72.3 \\
PEULI20 & 242.0 & 404.4 & -73.3 \\
PEUL240 & 240.1 & 404.3 & -73.0 \\
\hline
\end{tabular}

Abbreviations: $T_{\text {onset }}$, onset temperature of pyrolysis obtained from thermogravimetric analysis curves at $95 \%$ weight; $T_{p}$, peak pyrolytic temperature obtained from thermogravimetric analysis curves at $50 \%$ weight; $T_{g}$, glass transition temperature obtained from the first derivative of differntial scanning calometry curves; PEUS $30,60,120$, and 240 represents polyetherurethane containing 15, 30, 60, 120, and 240 ppm small-sized silver nanoparticles; PEUM I5, 30, 60, 120, and 240 represents polyetherurethane containing 15, 30, 60, 120, and 240 ppm medium-sized silver nanoparticles; PEUL 15, 30, 60, 120, and 240 represents polyetherurethane containing 30, 60, 120, and 240 ppm large-sized silver nanoparticles.

surface area in smaller AgNPs may increase the opportunity for interaction with the bacterial surface and promote a stronger antibacterial effect. ${ }^{19}$ On the other hand, AgNPs was reported to have significant cytotoxicity from many studies. ${ }^{6,8}$ The cytotoxicity was found at $10 \mathrm{ppm}$ AgNPs for a liver or neuron cell line after 24 hours of culture. In our study, comparable cytotoxicity at $10 \mathrm{ppm}$ was detected only for small-sized AgNPs. A similar finding was reported for macrophages. ${ }^{15}$ Therefore, the particle size may play a critical role in the cytotoxicity of AgNPs.

Even without a crosslinker, the tensile strength of PEU ionomer was obviously higher than that of PSU, by about twofold. This meant that PEU was a tougher material and a more durable elastomer than PSU. Therefore, PEU synthesized in this study should be more advantageous when used in various applications, including as a cardiovascular biomaterial. Although cell proliferation on PEU was slightly lower than that on PSU, this could be improved by addition of AgNPs, as also shown in this study. PSU requires reacting with a crosslinker before fabrication into the final product. After synthesis, PEU was in the form of an emulsion, and could be directly cast into films just like the conventional solvent-borne polyurethane. Compared with the conventional polyurethane, waterborne PEU still had lower tensile strength but was adequate for most biomedical applications. The cellular attachment and proliferation were better than those of the conventional polyurethane.

The changes in microstructure and AgNPs dispersion in PEU were associated with the thermal stability of PEU-Ag. When AgNPs were added into PEU, the values of three thermal parameters $\left(\mathrm{T}_{\mathrm{g}} / \mathrm{T}_{\mathrm{p}} / \mathrm{T}_{\text {onset }}\right)$ were changed slightly, suggesting the bulk structure was only slightly altered. The most significant increase in $\mathrm{T}_{\mathrm{p}}$ and decrease in $\mathrm{T}_{\mathrm{g}}$ were observed at $30 \mathrm{ppm}$ for PEUS, $60 \mathrm{ppm}$ for PEUM, and $15 \mathrm{ppm}$ for PEUL. These were probably the critical concentrations for dispersion of AgNPs. Beyond these concentrations, aggregation of AgNPs may have occurred, which led to deterioration of the positive effect.

Opposite to the slight effect on the bulk structure, the addition of AgNPs in different sizes and concentrations influenced the surface microstructure of PEU significantly. The better

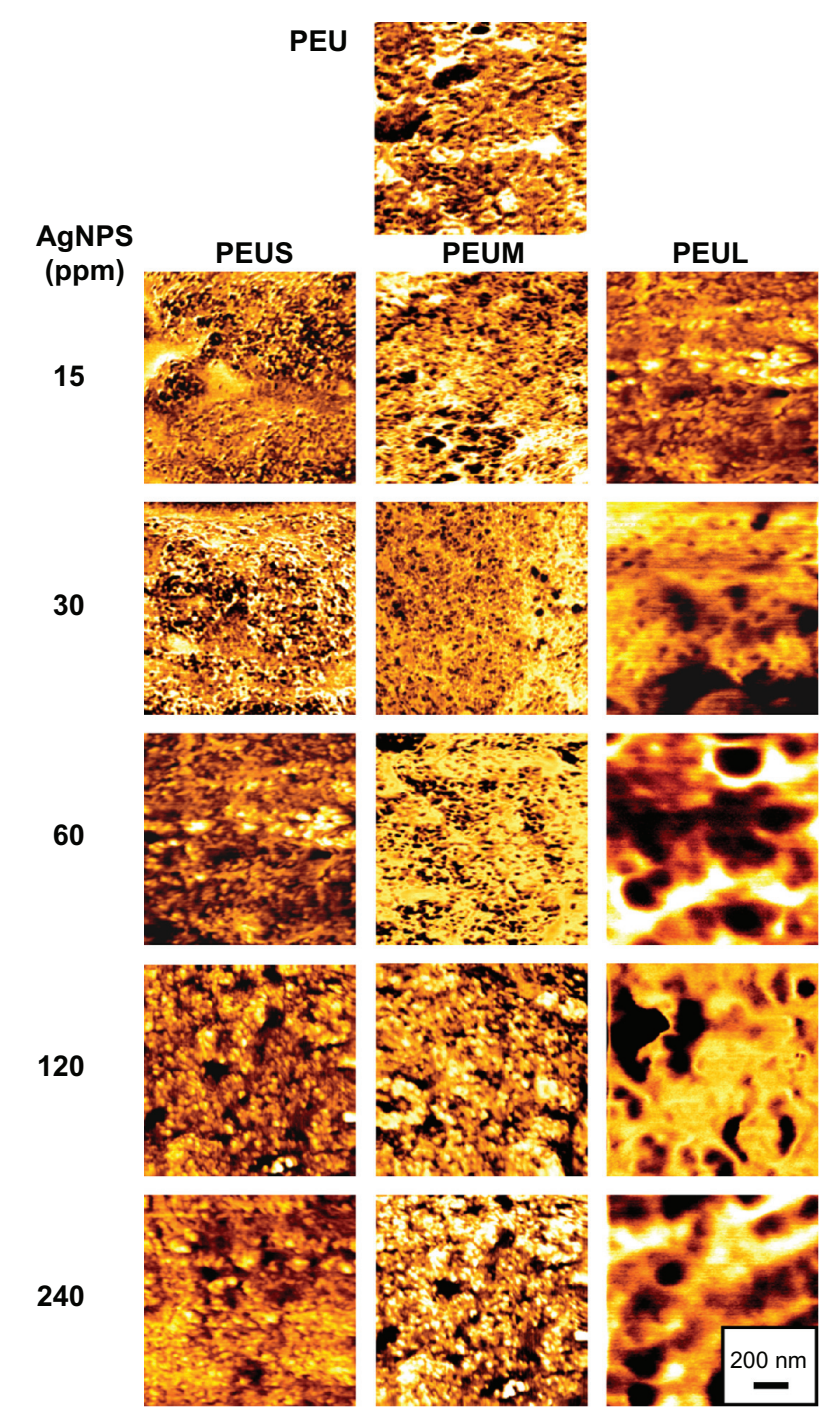

Figure 5 Atomic force microscopy phase images of polyetherurethane-silver nanocomposites containing different sizes and concentrations. 
Table 3 The values of surface zeta potential of polyetherurethane containing silver nanoparticles of different sizes

\begin{tabular}{llll}
\hline & \multicolumn{2}{l}{ Surface zeta potential $(\mathbf{m V})$} & \\
\cline { 2 - 3 } Polyetherurethane & $-15.55 \pm 1.14$ & PEUM & PEUL \\
\hline Silver concentration $(\mathbf{p p m})$ & PEUS & $-18.5 \pm 0.85$ & $-17.04 \pm 0.94$ \\
\hline 15 & $-14.11 \pm 1.41$ & $-21.22 \pm 2.12$ & $-16.83 \pm 0.53$ \\
30 & $-13.55 \pm 0.45$ & $-21.18 \pm 1.11$ & $-19.76 \pm 1.66$ \\
60 & $-18.19 \pm 0.92$ & $-20.40 \pm 2.65$ & $-19.64 \pm 0.90$ \\
120 & $-17.95 \pm 0.62$ & $-20.07 \pm 2.17$ & $-18.89 \pm 0.89$ \\
240 & $-17 \pm 0.26$ &
\end{tabular}

Abbreviations: PEUS, polyetherurethane mixed with small-sized silver nanoparticles; PEUM, polyetherurethane mixed with medium-sized silver nanoparticles; PEUL, polyetherurethane mixed with large-sized silver nanoparticles.

dispersion of soft domain and more delicate nanostructure were observed on the surface of PEUS $30 \mathrm{ppm}$, PEUM $60 \mathrm{ppm}$, and PEUL $15 \mathrm{ppm}$. The concentration-dependent
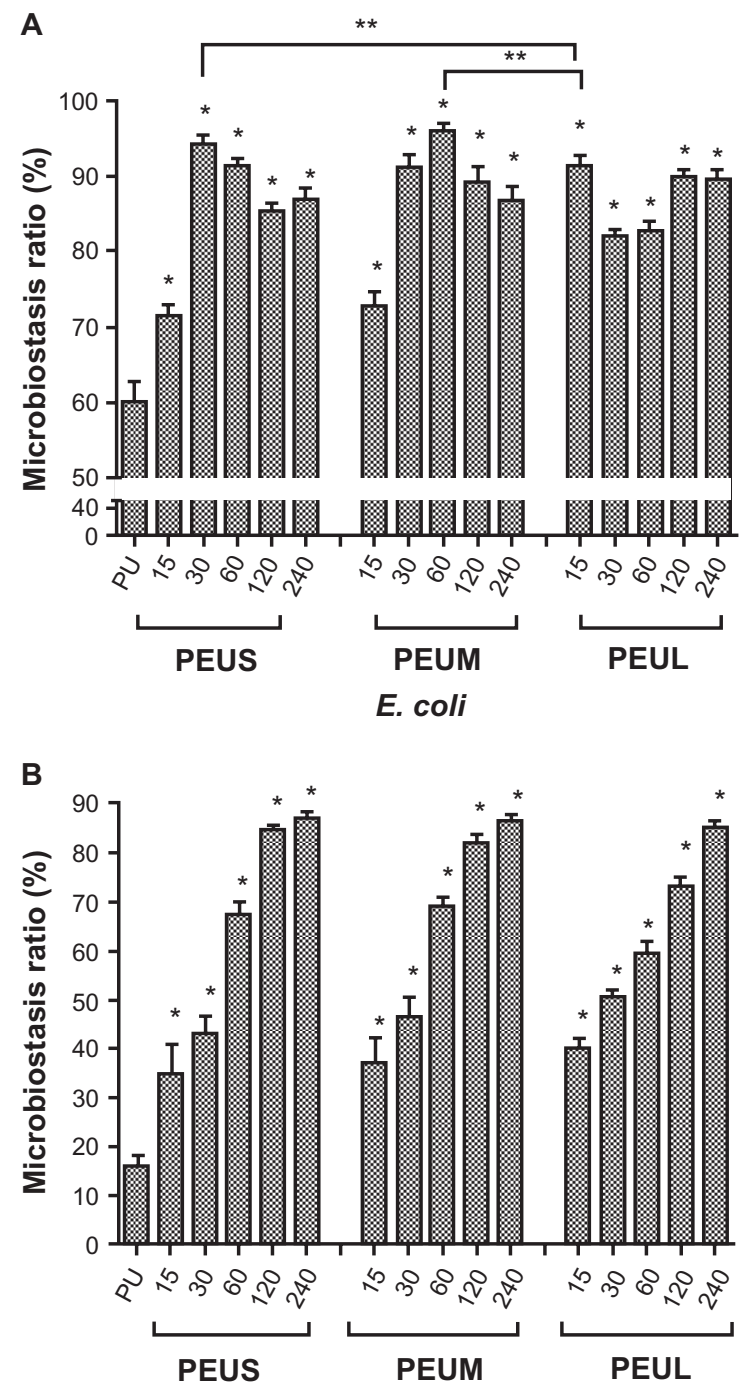

S. aureus

Figure 6 Microbiostatic effects against A) Escherichia coli and B) Staphylococcus aureus by polyetherurethane-silver nanocomposites containing silver nanoparticles of different sizes and concentrations after 24 hours.

Notes: $* P<0.05$, greater than polyetherurethane; **greater than PEULI5.

Abbreviation: PEULI5 polyetherurethane containing 15 ppm large-sized silver nanoparticles. influence of AgNPs on the surface of PEU was in a similar trend to that in the bulk but was more remarkable. Based on the previous study, ${ }^{15}$ the zeta potential of the particles was the largest for Ag-M $(15.43 \pm 2.72 \mathrm{mV})$, followed by Ag-S $(13.69 \pm 0.25 \mathrm{mV})$ and Ag-L $(5.35 \pm 1.26 \mathrm{mV})$. Particles with the more positive zeta potential are more stable and do not easily aggregate. Therefore, the upper limit of AgNP dispersion in PEU was probably ranked in the order of PEUM $>$ PEUS $>$ PEUL. Furthermore, the difference in particle size between Ag-S and Ag-M was small, so the dispersion largely depended on their zeta potential. Ag-L had a larger size and aggregated more easily (at $15 \mathrm{ppm}$ ). According to the previous study, ${ }^{20} \mathrm{AgNPs}$ influenced the distribution of hard segment (light) and soft (dark) segment domains of the polyurethane matrix. The optimal concentration of AgNPs was thus highly associated with their dispersion (nonaggregated state) in the polymer matrix. On the other hand, the largest microphase separation in the PEU ionomer occurred at $60 \mathrm{ppm}$ of medium-sized AgNPs, while in PSU it was at $30 \mathrm{pm} .{ }^{20}$ This may be attributed to the extra charges on the PEU ionomer, which contributed to the binding capacity for AgNPs.

The values of surface zeta potential were more negative for PEU-Ag nanocomposites compared with that of PEU. Ferraz et $\mathrm{al}^{21}$ indicated that zeta potential shifts towards negative charges resulted in a higher rate of cell proliferation and increased functional activity for human osteoblastic bone marrow-derived cells. The highest cell proliferation on PEUM $60 \mathrm{ppm}$ in this study also corresponded to the most negatively surface zeta potential, although the zeta potential for all nanocomposites was relatively close.

The plain PEU possessed certain antibacterial activity because of its amine group. The amine group can contact the bacterial surface to inhibit growth. ${ }^{22}$ The antibacterial activity of PEU-Ag nanocomposites was different from that of AgNPs. In addition, the antibacterial activity exhibited a dose-dependent increase for $S$. aureus, but not for E. coli. Generally, AgNPs had a better antibacterial effect for $E$. coli 
A

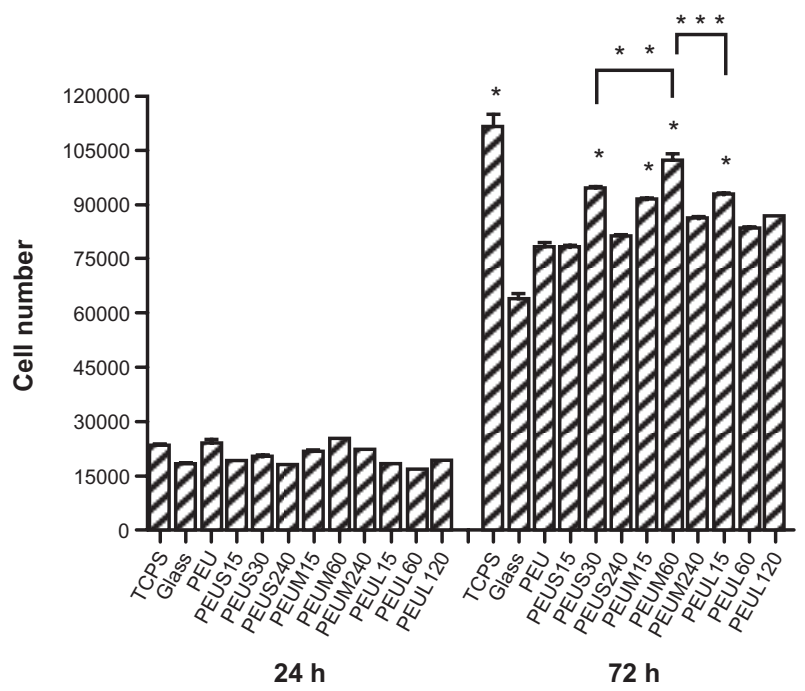

B

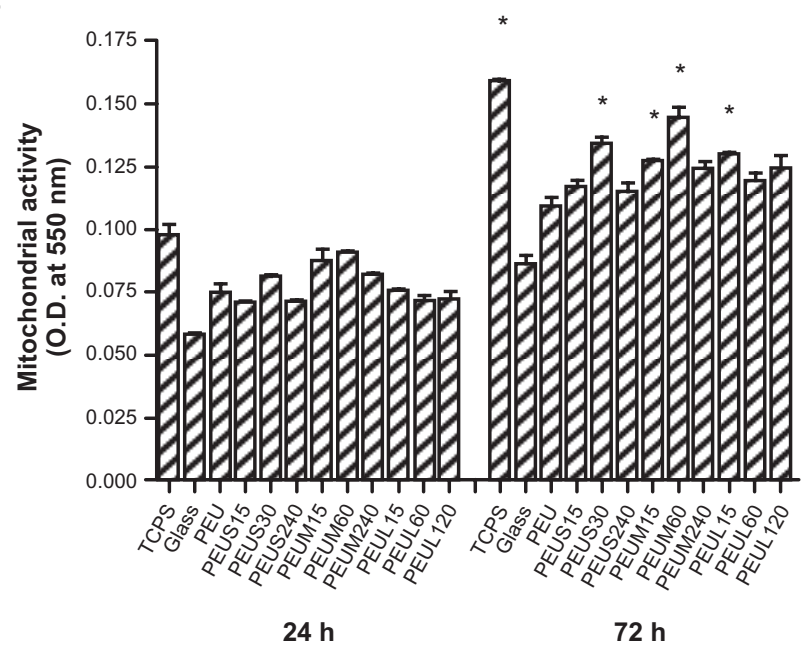

Figure 7 A) Number and B) mitochondrial activity of endothelial cells grown on polyetherurethane-silver.

Notes: $* P<0.05$ relative to polyetherurethane; $* * P<0.05$, $* * * P<0.05$

than for $S$. aureus. ${ }^{18}$ The maximal antibacterial activity against E. coli was observed in PEUS 30 ppm, PEUM $60 \mathrm{ppm}$, and PEUL15 ppm, corresponding to the respective concentration for the maximal microphase separation for each group. Therefore, the antibacterial effect of nanocomposites against $E$. col $i$ seemed to be related to the dispersion of AgNPs in PEU instead of the amount of AgNPs. This suggested that the only the nonaggregated well dispersed AgNPs exposed on the surface of PEU may inhibit the growth against $E$. coli efficiently. On the other hand, the mechanism of growth inhibition against $S$. aureus appeared to be different. The correlation of antibacterial activity with maximal phase separation/dispersion of AgNPs was absent for $S$. aureus. The maximal antibacterial activities against
A

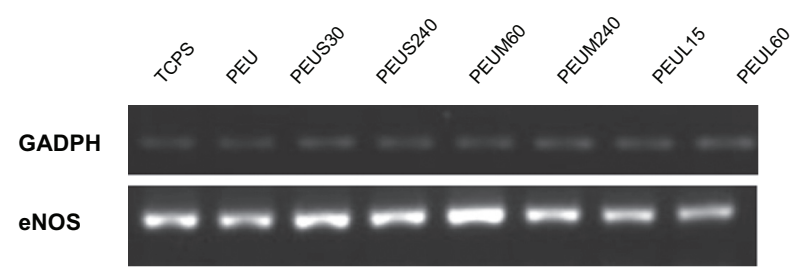

B

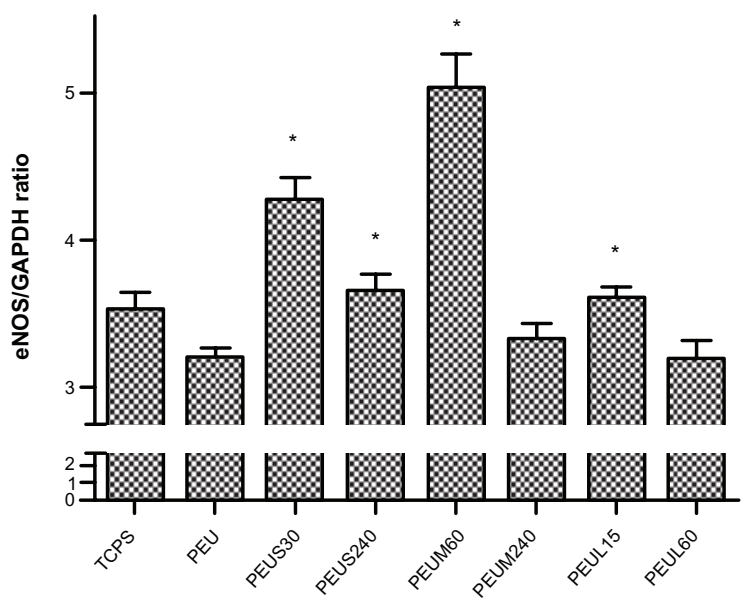

Figure 8 A) The expression of endothelial nitric oxide synthase gene and B) semiquantification of endothelial nitric oxide synthase/GAPDH ratio for endothelial cells cultured on the materials for three days.

Note: $* P<0.05$ relative to polyetherurethane.

S. aureus were the highest at the concentration of $240 \mathrm{ppm}$ for AgNPs of three different sizes. We assumed that, in addition to the exposed AgNPs on the surface, a higher concentration of AgNPs in PEU must be applied to release more free silver ion from the polymer matrix to inhibit the growth of $S$. aureus.

The proliferation of endothelial cells at 72 hours for each group of PEU-Ag nanocomposites was strongly associated with microphase separation. The highest proliferation was observed in PEUM 60 ppm among all groups. A previous study reported best cell compatibility at $30 \mathrm{ppm}$ of Ag-M for a different PU (ie, PSU in this study). ${ }^{20}$ The difference may be caused by the binding between AgNPs and charged functional groups (ionomers) of PEU as mentioned. Again, expression of the eNOS gene in endothelial cells was further upregulated by the nanocomposites, especially for PEUS $30 \mathrm{ppm}$ and PEUM $60 \mathrm{ppm}$. It has been reported that the addition of gold nanoparticles in PSU induced eNOS gene expression and promoted cell proliferation and migration. ${ }^{13}$ Therefore, a greater level of eNOS expression was a highly desired characteristic of the nanocomposites, but could only be achieved when AgNPs were at the optimal sizes and concentrations. 
Table 4 The number of adhered human blood platelets on the surface of polyetherurethane-silver nanocomposites after incubation for one hour

\begin{tabular}{llll}
\hline $\begin{array}{l}\text { Concentration of } \\
\text { AgNPs }(\mathrm{ppm})\end{array}$ & $\begin{array}{l}\text { PEUS } \\
\left(\times 10^{6}\right)\end{array}$ & $\begin{array}{l}\text { PEUM } \\
\left(\times 10^{6}\right)\end{array}$ & $\begin{array}{l}\text { PEUL } \\
\left(\times 10^{6}\right)\end{array}$ \\
\hline 0 & $11.0 \pm 0.4$ & & \\
15 & $7.9 \pm 0.4$ & $7.5 \pm 0.3^{*}$ & $7.9 \pm 0.3^{*}$ \\
30 & $7.8 \pm 0.2^{*}$ & $6.4 \pm 0.4^{*}$ & $9.1 \pm 0.3$ \\
60 & $6.1 \pm 0.4^{*}$ & $8.0 \pm 0.4^{*}$ & $9.9 \pm 0.6$ \\
120 & $8.3 \pm 0.3^{*}$ & $8.4 \pm 0.7^{*}$ & $7.8 \pm 0.6^{*}$ \\
240 & $8.5 \pm 0.3^{*}$ & $8.9 \pm 0.3$ & $8.2 \pm 0.6^{*}$ \\
\hline
\end{tabular}

Note: $* P<0.05$ relative to polyetherurethane.

Abbreviations: AgNPs, silver nanoparticles; PEUS, polyetherurethane mixed with small-sized silver nanoparticles; PEUM, polyetherurethane mixed with mediumsized silver nanoparticles; PEUL, polyetherurethane mixed with large-sized silver nanoparticles.

Platelet adhesion is a very important index for cardiovascular applications. Monocyte activation triggers the inflammation cascade and can reflect the inflammatory response. The nanocomposites in this study were found to reduce platelet adhesion. Monocyte activation was reduced only in those nanocomposites with better surface microphase separation, again suggesting the dominant role of surface nanostructure on the biocompatibility of polyurethane polymers.

Overall, PEU nanocomposites with the medium-sized AgNPs $(\sim 5 \mathrm{~nm})$ showed the best performance in all aspects. The inclusion of AgNPs with a smaller $(\sim 3 \mathrm{~nm})$ or larger $(\sim 24 \mathrm{~nm})$ average size was not as effective. This suggested that the size of AgNPs was critical to their positive effect in nanocomposites, including biocompatibility and antibacterial properties. Because $5 \mathrm{~nm}$ was very close to the typical characteristic length of polyurethane, we hypothesized that nanoparticles at the comparable size could manipulate the hard-soft interface and change the two-phase morphology of polyurethane and hence the performance more effectively. The results of this study also indicate that the antibacterial effect of AgNPs embedded in a polymer depends on the size, concentration, and distribution of AgNPs in the polymer matrix.

\section{Conclusion}

In this study, AgNPs of smaller sizes had better antibacterial properties but higher cytotoxicity. Nanocomposites from a new polyurethane ionomer and AgNPs had no cytotoxicity. The nanocomposites had excellent antibacterial activity. Nanocomposites with $60 \mathrm{ppm}$ of medium size $(5 \mathrm{~nm})$ AgNPs had the best antibacterial activity, biocompatibility, and eNOS gene expression. We believe that this was due to the effectiveness of these AgNPs in producing a delicate two-phase nanostructure on their surface. The good dispersion of AgNPs in the structure allowed exposed nonaggregated AgNPs to the surface and cause growth inhibition of $E$. coli. The delicate nanostructure also contributed to the biocompatibility of the polyurethane nanocomposites.

\section{Acknowledgment}

The research was sponsored by a grant (98-EC-17-A17-S1-111) from the Ministry of Economic Affairs, Executive Yuan, Taiwan, Republic of China.

\section{Disclosure}

The authors report no conflicts of interest in this work.

\section{References}

1. Zuhuang J. Bactericidal nanosilver cloth and its making process and use. Patent number CN 1387700. 2003.

2. Lee HJ, Yeo SY, Jeong SH. Antibacterial effect of nanosized silver colloidal solution on textile fabrics. J Mat Sci. 2003;38(10):2199-2204.

3. Jain P, Pradeep T. Potential of silver nanoparticle-coated polyurethane foam as an antibacterial water filter. Biotechnol Bioeng. 2005;90(1): 59-63.

4. Alt V, Bechert T, Steinrücke P, et al. Nanoparticulate silver. A new antimicrobial substance for bone cement. Orthopade. 2004;33(8): 885-892.

5. Foldbjerg R, Dang DA, Autrup H. Cytotoxicity and genotoxicity of silver nanoparticles in the human lung cancer cell line, A549. Arch Toxicol. 2010 April 29. [Epub ahead of print].

6. Hussain SM, Hess KL, Gearhart JM, Geiss KT, Schlager JJ. In vitro toxicity of nanoparticles in BRL 3A rat liver cells. Toxicol In Vitro. 2005;19(7):975-983.

7. Nallathamby PD, Xu XH. Study of cytotoxic and therapeutic effects of stable and purified silver nanoparticles on tumor cells. Nanoscale. 2010;2(6):942-952.

8. Hussain SM, Javorina AK, Schrand AM, Duhart HM, Ali SF, Schlager JJ The interaction of manganese nanoparticles with PC-12 cells induces dopamine depletion. Toxicol Sci. 2006;92(2):456-463.

9. Barrere M, Landfester K. High molecular weight polyurethane and polymer hybrid particles in aqueous miniemulsion. Macromolecules 2003;36(14):5119-5125.

10. Cao X, Lee LJ, Widya T, Macosko C. Polyurethane/clay nanocomposites foams: Processing, structure and properties. Polymer. 2005;46(3):775-783.

11. Decker C, Vataj R, Louati A. Synthesis of acrylic polymer networks by electroinitiated polymerization. Prog Org Coat. 2004;50(4):263-268.

12. Modesti M, Lorenzetti A. An experimental method for evaluating isocyanate conversion and trimer formation in polyisocyanate-polyurethane foams. Eur Poly J. 2001;37(5):949-954.

13. Hung HS, Wu CC, Chien S, Hsu SH. The behavior of endothelial cells on polyurethane nanocomposites and the associated signaling pathways Biomaterials. 2009;30(8):1502-1511.

14. Chou CW, Hsu SH, Chang H, Tseng SM, Lin HR. Enhanced thermal and mechanical properties and biostability of polyurethane containing silver nanoparticles. Polym Degrad Stab. 2006;91(5): 1017-1024.

15. Yen HJ, Hsu SH, Tsai CL. Cytotoxicity and immunological response of gold and silver nanoparticles of different sizes. Small. 2009;5(13): 1553-1561.

16. Hsu SH, Tang CM, Tseng HJ. Gold nanoparticles induce surface morphological transformation in polyurethane and affect the cellular response. Biomacromolecules. 2008;9(1):241-248. 
17. Hsu S, Kao YC, Lin ZC. Enhanced biocompatibility in biostable poly(carbonate) urethane. Macromol Biosci. 2004;4(4):464-470.

18. Kim JS, Kuk E, Yu KN, et al. Antimicrobial effects of silver nanoparticles. Nanomedicine. 2007;3(1):95-101.

19. Martínez-Castañón GA, Niño-Martínez N, Martínez-Gutierrez F, Martínez-Mendoza JR, Ruiz F. Synthesis and antibacterial activity of silver nanoparticles with different sizes. J Nanopart Res. 2008; 10(8): 1343-1348.

20. Hsu SH, Tseng HJ, Lin YC. The biocompatibility and antibacterial properties of waterborne polyurethane-silver nanocomposites. Biomaterials. 2010;31(26):6796-6808.
21. Ferraz MP, Fernandes MH, Santos JD, Monteiro FJ. HA and doublelayer HA-P2O5/CaO glass coatings: Influence of chemical composition on human bone marrow cells osteoblastic behavior. J Mater Sci Mater Med. 2001;12(7):629-638.

22. Atef El-Sayed A, El Gabry LK, Allam OG. Application of prepared waterborne polyurethane extended with chitosan to impart antibacterial properties to acrylic fabrics. J Mater Sci Mater Med. 2010;21(2): 507-514.

\section{Publish your work in this journal}

The International Journal of Nanomedicine is an international, peerreviewed journal focusing on the application of nanotechnology in diagnostics, therapeutics, and drug delivery systems throughout the biomedical field. This journal is indexed on PubMed Central, MedLine, CAS, SciSearch ${ }^{\circledR}$, Current Contents ${ }^{\circledR} /$ Clinical Medicine,
Journal Citation Reports/Science Edition, EMBase, Scopus and the Elsevier Bibliographic databases. The manuscript management system is completely online and includes a very quick and fair peer-review system, which is all easy to use. Visit http://www.dovepress.com/ testimonials.php to read real quotes from published authors. 